\title{
A DETERMINANTAL INEQUALITY FOR PROJECTORS IN A UNITARY SPACE ${ }^{1}$
}

\author{
D. ž. DJOKOVIĆ
}

Abstract. Let $V$ be a finite dimensional unitary space and $V=V_{1}+\cdots+V_{k}$ a direct decomposition. Let $\boldsymbol{P}_{i}$ be the orthogonal projector in $V$ with range $V_{i}$. If $A=P_{1}+\cdots+P_{k}$ we prove that $0<\operatorname{det}(A) \leqq 1$ and $\operatorname{det}(\boldsymbol{A})=1$ if and only if $V=V_{1}+\cdots+V_{k}$ is an orthogonal decomposition.

Let $N_{i}(1 \leqq i \leqq k)$ be a normal operator in $V$, of rank $r_{i}$. Assume that $N=\sum_{i=1}^{k} N_{i}$ has rank $r=r_{1}+\cdots+r_{k} \leqq n=\operatorname{dim} V$. If the nonzero eigenvalues of $\boldsymbol{N}$ (counting multiplicities) are the same as the nonzero eigenvalues of all $\boldsymbol{N}_{i}(1 \leqq i \leqq k)$ together, then $\boldsymbol{N}_{i} \boldsymbol{N}_{j}=0$ for $i \neq j$. This generalizes a recent result of $L$. Brand.

I. Introduction. The following theorem was proved recently by L. Brand [1]:

TheOREM 1. Let $A$ and $B$ be real $n \times n$ symmetric matrices with $\lambda_{1}, \cdots, \lambda_{r}, 0, \cdots, 0$ and $0, \cdots, 0, \lambda_{r+1}, \cdots, \lambda_{n}\left(\lambda_{i} \neq 0,1 \leqq i \leqq n\right)$ as eigenvalues, respectively. If $A+B$ has eigenvalues $\lambda_{1}, \lambda_{2}, \cdots, \lambda_{n}$ then $A B=0$.

One of the aims of this paper is to generalize this theorem. We shall prove that the assertion remains valid when $A$ and $B$ are complex normal $n \times n$ matrices. Moreover, we shall prove that an analogous result holds for sums of several normal matrices (Theorem 3 ).

In this paper $V$ denotes $n$-dimensional complex unitary space with scalar product $(x, y)$. Our Theorem 2 gives a necessary and sufficient condition for a direct sum $V=V_{1}+\cdots+V_{k}$ to be an orthogonal direct sum.

II. Sum of projectors. We begin by proving three lemmas:

LEMMA 1. Let $A$ be an operator in $V$ such that:

(i) all eigenvalues of $A$ have modulus one,

(ii) $\|A x\| \leqq\|x\|$ for all $x \in V$.

Then $A$ is a unitary operator.

Proof. In view of (i), it is sufficient to show that $A$ is normal.

Received by the editors December 1, 1969.

AMS 1969 subject classifications. Primary 1570, 1558; Secondary 4615.

Key words and phrases. Unitary space, orthogonal decomposition, eigenvalues, eigenvectors, projector, normal operator, hermitian positive definite operator.

1 This work was supported in part by N.R.C. Grant A-5285. 
We claim that the eigenvectors of $A$ span $V$. If this is false, there exists an eigenvector $x$ of $A$ such that $A x=\lambda x,\|x\|=1, A y=\lambda y+x$ for some $y \in V$. For $z=y-(y, x) x$ we have $A z=\lambda z+x,\|A z\|^{2}=\|z\|^{2}+1$ which contradicts (ii). This proves that our claim is true.

We shall prove that $A$ is normal by showing that $A x=\lambda x, A y=\mu y$, $\lambda \neq \mu$ imply that $(x, y)=0$. Assume that $(x, y) \neq 0$. Replacing $x$ by $\theta x$ where $\theta$ is a suitable nonzero complex number, we can further assume that the real part of $\left(\lambda_{\bar{\mu}}-1\right)(x, y)$ is positive. Then

$$
\begin{aligned}
\|A(x+y)\|^{2}-\|x+y\|^{2} & =\|\lambda x+\mu y\|^{2}-\|x+y\|^{2} \\
& =2 \operatorname{Re}\{(\lambda \bar{\mu}-1)(x, y)\}>0
\end{aligned}
$$

contradicts (ii). Therefore we must have $(x, y)=0$.

The lemma is proved.

Lemma 2. Let $V=V_{1}+V_{2}$ be a direct sum, $V_{i} \neq 0, i=1$, 2. Let $\boldsymbol{P}_{i}$ $(i=1,2)$ be the orthogonal projector in $V$ with range $V_{i}$ and

$$
P_{12}: V_{1} \rightarrow V_{2} \text { and } P_{21}: V_{2} \rightarrow V_{1}
$$

be the restrictions of $P_{2}$ and $P_{1}$, respectively. Then

$$
\operatorname{det}\left(I_{2}-P_{12} P_{21}\right) \leqq 1
$$

where $I_{2}: V_{2} \rightarrow V_{2}$ is the identity operator. The equality holds in (1) if and only if $V_{1}$ is orthogonal to $V_{2}$.

Proof. Since $P_{1}$ and $P_{2}$ are hermitian positive semidefinite the same is true for $B=P_{1}+P_{2}$. If $x \in V$ and $B x=P_{1} x+P_{2} x=0$ then $P_{1} x=P_{2} x=0$ since $V=V_{1}+V_{2}$ is a direct sum. We infer that $x$ is orthogonal to $V_{1}$ and $V_{2}$ and consequently $x=0$. Hence, $B$ is in fact positive definite. If we choose bases in $V_{1}$ and $V_{2}$ they form a base of $V$. In such a base the matrix of $B$ is

$$
B=\left(\frac{I_{1}}{P_{12}} \mid \frac{P_{21}}{I_{2}}\right)
$$

where $I_{1}, I_{2}, P_{12}, P_{21}$ are matrices of $I_{1}, I_{2}, P_{12}, P_{21}$, respectively $\left(I_{1}: V_{1} \rightarrow V_{1}\right.$ is the identity operator). We have

$$
\operatorname{det}(A)=\operatorname{det}\left(\frac{I_{1}}{P_{12}} \mid \frac{0}{I_{2}-P_{12} P_{21}}\right)=\operatorname{det}\left(\frac{I_{1}}{P_{12}} \mid \frac{P_{21}}{I_{2}}\right)=\operatorname{det}(B)>0 .
$$

If $x \in V_{2}$ then

$$
\begin{aligned}
\|A x\|^{2} & =(A x, A x)=\left(x-P_{2} P_{1} x, x-P_{2} P_{1} x\right) \\
& =\|x\|^{2}-\left(P_{2} P_{1} x, x\right)-\left(x, P_{2} P_{1} x\right)+\left\|P_{2} P_{1} x\right\|^{2} \\
& =\|x\|^{2}-2\left\|P_{1} x\right\|^{2}+\left\|P_{2} P_{1} x\right\|^{2}
\end{aligned}
$$


because $\left(P_{2} P_{1} x, x\right)=\left(P_{1} x, P_{2} x\right)=\left(P_{1} x, x\right)=\left\|P_{1} x\right\|^{2}$ and $\left(x, P_{2} P_{1} x\right)$ $=\left\|P_{1} x\right\|^{2}$. It follows that

$$
\|A x\|^{2} \leqq\|x\|^{2}-\left\|P_{1} x\right\|^{2}
$$

since $\left\|P_{1} x\right\|^{2}-\left\|P_{2} P_{1} x\right\|^{2} \geqq 0$. We infer that $|\lambda| \leqq 1$ for every eigenvalue $\lambda$ of $A$. Hence, $|\operatorname{det}(A)| \leqq 1$ which together with $\operatorname{det}(A)>0$ establishes (1).

If $\operatorname{det}(A)=1$ then every eigenvalue of $A$ must have modulus 1 . By Lemma 1 and (2), $\boldsymbol{A}$ is unitary. Now, (2) implies that $\boldsymbol{P}_{1} x=0$ for all $x \in V_{2}$, i.e., $V_{1}$ and $V_{2}$ are orthogonal to each other. The converse is trivial.

Lemma 3. Let $V=V_{1}+V_{2}$ be a direct sum, $V_{i} \neq 0, i=1,2$. Let $H$ be $a$ hermitian operator in $V$ with range $V_{1}$, such that its restriction $H_{1}$ to $V_{1}$ is positive definite, and let $P_{2}$ be the orthogonal projector in $V$ with range $V_{2}$. Then

$$
0<\operatorname{det}\left(\boldsymbol{H}+\boldsymbol{P}_{2}\right) \leqq \operatorname{det}\left(\boldsymbol{H}_{1}\right)
$$

The equality holds in (3) if and only if $V_{1}$ is orthogonal to $V_{2}$.

Proof. We fix bases in $V_{1}$ and $V_{2}$. They form together a basis of $V$. All matrices will be taken with respect to these bases. The matrix of $H+P_{2}$ has the form

$$
\left(\frac{H_{1}}{P_{12}} \mid \frac{H_{1} P_{21}}{I_{2}}\right)
$$

where:

$H_{1}$ is the matrix of $H_{1}$,

$P_{12}$ is the matrix of the orthogonal projector $P_{12}: V_{1} \rightarrow V_{2}$, $P_{21}$ is the matrix of the orthogonal projector $P_{21}: V_{2} \rightarrow V_{1}$, $I_{2}$ is the identity matrix.

Since

$$
\operatorname{det}\left(\frac{H_{1}}{P_{12}} \mid \frac{H_{1} P_{21}}{I_{2}}\right)=\operatorname{det}\left(\frac{H_{1}}{P_{12}} \mid \frac{0}{I_{2}-P_{12} P_{21}}\right)
$$

we get

$$
\operatorname{det}\left(\boldsymbol{H}+\boldsymbol{P}_{2}\right)=\operatorname{det}\left(\boldsymbol{H}_{1}\right) \cdot \operatorname{det}\left(\boldsymbol{I}_{2}-\boldsymbol{P}_{12} \boldsymbol{P}_{21}\right) .
$$

It remains to apply Lemma 2 .

Theorem 2. Let

$$
V=V_{1}+\cdots+V_{k}
$$


be a direct sum, $V_{i} \neq 0$. Let $P_{i}$ be the orthogonal projector in $V$ with range $V_{i}$ and let $A=P_{1}+\cdots+P_{k}$. Then $0<\operatorname{det}(A) \leqq 1$ and $\operatorname{det}(A)=1$ if and only if (4) is an orthogonal sum.

Proof. $A$ is hermitian positive definite (the argument is the same as for $B$ in the proof of Lemma 2). Hence, $\operatorname{det}(A)>0$.

We shall prove the remaining assertions of the theorem by induction on $k$. If $k=1$, they are trivially true. For $k>1$ we put

$$
U=V_{1}+\cdots+V_{k-1} \text {. }
$$

Let $\boldsymbol{Q}_{i}(1 \leqq i \leqq k-1)$ be the restrictions of $P_{i}$ to $U$ and $H_{1}=Q_{1}+\cdots$ $+Q_{k-1}$. By inductive hypothesis and the first part of the proof, we conclude that $0<\operatorname{det}\left(\boldsymbol{H}_{1}\right) \leqq 1$ and $\operatorname{det}\left(\boldsymbol{H}_{1}\right)=1$ if and only if (5) is an orthogonal sum. We can apply Lemma 3 with $H=P_{1}+\cdots+P_{k-1}$. It follows that $0<\operatorname{det}(A)=\operatorname{det}\left(H+P_{k}\right) \leqq \operatorname{det}\left(\boldsymbol{H}_{\mathbf{1}}\right) \leqq 1$.

If $\operatorname{det}(\boldsymbol{A})=1$ then $V_{k}$ and $U$ are orthogonal to each other by Lemma 3. Since (5) is also an orthogonal sum, the theorem is proved.

III. Sum of normal operators. Now we are able to prove the following:

TheOREM 3. Let $\boldsymbol{N}_{i}, 1 \leqq i \leqq k$, be normal operators in $V$ with eigenvalues

$$
\lambda_{1}^{(i)}, \cdots, \lambda_{r_{i}}^{(i)}, 0, \cdots, 0,
$$

where $\lambda_{j}^{(t)} \neq 0,1 \leqq j \leqq r_{i}, 1 \leqq i \leqq k$ and $r_{1}+\cdots+r_{k} \leqq n$. If the operator $N=N_{1}+\cdots+N_{k}$ has eigenvalues

$$
\lambda_{j}^{(i)}, \quad 1 \leqq i \leqq k, 1 \leqq j \leqq r_{i},
$$

and 0 with multiplicity $n-\left(r_{1}+\cdots+r_{k}\right)$ then the ranges of $\boldsymbol{N}_{i}$, $1 \leqq i \leqq k$, are orthogonal to each other, $\boldsymbol{N}$ is normal and $\boldsymbol{N}_{i} \boldsymbol{N}_{j}=\mathbf{0}$ for $i \neq j$.

Proof. Let $V_{i}$ be the range of $\boldsymbol{N}_{\boldsymbol{i}}$. Since

$$
\operatorname{rank} N=\sum_{i=1}^{k} \operatorname{rank} N_{i}
$$

we infer that the sum $U=V_{1}+\cdots+V_{k}$ is direct. Let $W$ be the orthogonal complement of $U$. Then $W$ is contained in the null space of each operator $\boldsymbol{N}_{i}$. Hence $W$ is the null space of $\boldsymbol{N}$.

Hence, without loss of generality, we can assume that $r_{1}+\ldots$ $+r_{k}=n$; i.e., that $N$ is nonsingular. 
Let $\boldsymbol{P}_{i}$ be the orthogonal projector in $V$ with range $V_{i}$. Let $\boldsymbol{P}_{j i}: V_{j}$ $\rightarrow V_{i}$ be the restriction of $P_{i}$ and $M_{i}: V_{i} \rightarrow V_{i}$ the restriction of $\boldsymbol{N}_{i}$. We fix a basis in each $V_{i}$. Since

$$
N=\sum_{i=1}^{k} N_{i}=\sum_{i=1}^{k} N_{i} P_{i}=\sum_{i=1}^{k} M_{i} P_{i}
$$

the matrix of $\boldsymbol{N}$ has the form

$$
N=\left(\begin{array}{cc}
M_{1} P_{11} & M_{1} P_{21} \cdots \\
M_{2} P_{12} & M_{2} P_{22} \\
\vdots &
\end{array}\right)
$$

where $M_{i}, P_{i j}$ are matrices of $M_{i}, P_{i j}$.

The conditions on eigenvalues of $\boldsymbol{N}_{i}$ and $\boldsymbol{N}$ imply that

$$
\operatorname{det}(\boldsymbol{N}-\lambda I)=\prod_{i=1}^{k} \operatorname{det}\left(\boldsymbol{M}_{\boldsymbol{i}}-\lambda I_{i}\right)
$$

For $\lambda=0$ we $g e t \operatorname{det}(N)=\prod_{i=1}^{k} \operatorname{det}\left(M_{i}\right)$. Since

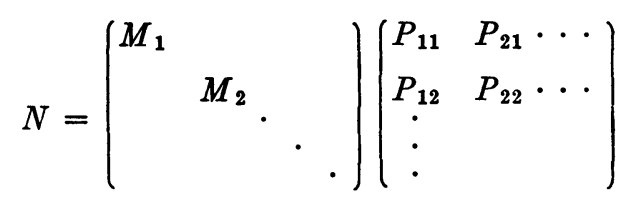

we infer that

$$
\operatorname{det}\left(\begin{array}{cc}
P_{11} & P_{21} \cdots \\
P_{12} & P_{22} \cdots \\
\vdots & \vdots
\end{array}\right)=1
$$

This is equivalent to

$$
\operatorname{det}\left(P_{1}+\cdots+P_{k}\right)=1 .
$$

Theorem 2 implies that $V=V_{1}+\cdots+V_{k}$ is an orthogonal direct sum.

The remaining assertions follow easily.

\section{REFERENCES}

1. L. Brand, On the product of singular symmetric matrices, Proc. Amer. Math. Soc. 22 (1969), 377. MR $39 \# 4183$.

University of Waterloo, Waterloo, Ontario, Canada 